\title{
Prescripciones de diuréticos de asa potencialmente inapropiadas en ancianos
}

\section{Jorge E. Machado-Alba ${ }^{a, b, *}$, Claudia Giraldo-Giraldo ${ }^{a, b}$ y Sivia Fernanda Urbano-Garzón ${ }^{a, b}$}

\section{a Grupo de Investigación en Farmacoepidemiología y Fármacovigilancia. Departamento de Ciencias Básicas, Facultad de Ciencias} de la Salud, Universidad Tecnológica de Pereira, Pereira, Colombia

${ }^{\mathrm{b}}$ Audifarma S.A. Colombia, Pereira, Colombia

Recibido el 13 de abril de 2016; aceptado el 12 de julio de 2016

Disponible en Internet el 22 de septiembre de 2016

\author{
PALABRAS CLAVE \\ Hipertensión arterial; \\ Atención primaria; \\ Farmacología; \\ Diurético
}

\begin{abstract}
Resumen
Objetivo: Identificar la prescripción potencialmente inadecuada de diuréticos de asa como monoterapia de primera línea en hipertensión arterial de pacientes mayores de 65 años en Colombia.

Métodos: Estudio cuasiexperimental, prospectivo, antes y después, sin grupo control, mediante una intervención educativa dirigida a prescriptores de pacientes mayores de 65 años con diagnóstico de hipertensión arterial en manejo con furosemida en monoterapia, afiliados al régimen pago del Sistema de Salud de Colombia entre el $1^{\circ}$. de enero y el 30 de agosto de 2014 . Luego de la identificación de los pacientes de una base de datos poblacional de dispensación de medicamentos y de la intervención se midió el cambio en la formulación.

Resultados: De un total de 453.017 sujetos en tratamiento antihipertensivo se identificaron 160 pacientes con diagnóstico de hipertensión arterial tratados con furosemida en monoterapia, con una edad media de $82,7 \pm 9,1$ años. Se realizaron 76 actividades educativas sobre los médicos y se consiguió modificar la terapia en 67 pacientes $(41,8 \%$ de los intervenidos), donde el 6,8\% suspendió el diurético, pero inició un nuevo antihipertensivo, 13,1\% agregó un antihipertensivo y $21,8 \%$ lo suspendió definitivamente. Ser anciano entre 65 y 74 años se asoció con mayor probabilidad de éxito en la intervención (OR: 2,2; IC95\%:1,010-4,860; $\mathrm{p}<0,001$ ).

Conclusiones: Una pequeña proporción de pacientes mayores de 65 años son tratados con furosemida en monoterapia antihipertensiva, pero las estrategias educativas y de seguimiento de las prescripciones pueden reducir su uso inapropiado. Es importante desarrollar herramientas que incentiven al médico a evitar prescripciones menos eficaces y seguras.

(c) 2016 Sociedad Colombiana de Cardiología y Cirugía Cardiovascular. Publicado por Elsevier España, S.L.U. Este es un artículo Open Access bajo la licencia CC BY-NC-ND (http:// creativecommons.org/licenses/by-nc-nd/4.0/).
\end{abstract}

\footnotetext{
* Autor para correspondencia.

Correos electrónicos: machado@utp.edu.co, jormach66@hotmail.com (J.E. Machado-Alba).
} 


\section{KEYWORDS}

Arterial hypertension;

Primary care;

Pharmacology;

Diuretic

\section{Potentially inappropriate loop diuretic prescriptions in the elderly}

\begin{abstract}
Motivation: To identify the potentially inappropriate prescription of loop diuretics as a first-line monotherapy in arterial hypertension of patients over 65 years old in Colombia.

Methods: Quasiexperimental, prospective, before and after study, without a control group, by means of an educational intervention aimed at prescriptors of patients over 65 years old with the diagnosis of arterial hypertension managed with furosemide in monotherapy, affiliated to the Colombian Health System between January $1^{\text {st }}$ and August $30^{\text {th }} 2014$. After identifying the patients in a population database of medication dispensation and after the intervention, changes in the formulation were measured.

Results: Out of a total of 453,017 individuals taking antihypertensives, 160 patients were identified with arterial hypertension being treated with furosemide in monotherapy, with an average age of $82.7 \pm 9.1$ years. 76 educational activities were carried out with physicians and therapy could be modified in 67 patients (47.8\% of intervention participants), where $6.8 \%$ discontinued the diuretic, but initiated a new antihypertensive, $13.1 \%$ added an antihypertensive and $21.8 \%$ definitely suspended the treatment. Being between 65 and 74 years old was associated to a higher probability of success of the intervention (OR: 2.2; cl 95\%: 1.010-4.860; $p<0.001$ ). Conclusions: A small proportion of patients over 65 years of age were treated with furosemide as antihypertensive monotherapy, but educational strategies and monitorisation of prescriptions may reduce their inappropriate use. It is important to develop tools to motivate the physician to avoid less efficient and safe prescriptions.

(c) 2016 Sociedad Colombiana de Cardiología y Cirugía Cardiovascular. Published by Elsevier España, S.L.U. This is an open access article under the CC BY-NC-ND license (http:// creativecommons.org/licenses/by-nc-nd/4.0/).
\end{abstract}

\section{Introducción}

Se estima que las reacciones adversas a medicamentos (RAM) son responsables de hasta un $30 \%$ de los ingresos hospitalarios en los pacientes mayores, siendo la prescripción potencialmente inapropiada (PPI) de fármacos y la mala monitorización de tratamientos prescritos las principales causales. Los criterios Stopp y Start (Screening Tool of Older Person's potentially inappropriate Prescriptions/Screening Tool to Alert doctors to the Right, i.e. appropriate, indicated Treatment) detectan tratamientos farmacológicos potencialmente inapropiados en personas mayores de 65 años, incluidos errores por omisión en la formulación de fármacos que probablemente benefician al paciente y la prescripción de medicamentos inadecuados; entre estos últimos se encuentra el uso de diuréticos de asa como monoterapia de primera línea en hipertensión arterial, lo cual está desaconsejado dado que existen alternativas más seguras y efectivas $^{1,2}$.

Respecto al tratamiento antihipertensivo, la guía basada en la evidencia para el manejo de la hipertensión arterial en adultos 2014, del Report From the Panel Members Appointed to the Eight Joint National Committee, recomienda los siguientes fármacos: diurético tiazídico, bloqueadores de los canales de calcio, inhibidores de la enzima convertidora de angiotensina (IECA), o bloqueadores del receptor de angiotensina (BRA-II) ${ }^{3}$. De hecho, no se mencionan y por tanto no se recomiendan los diuréticos de asa para el manejo de la hipertensión arterial, lo que hace de suma importancia evaluar su prescripción. En efecto, los criterios Stopp y Start pretenden educar al prescriptor en la optimización de la terapéutica en los pacientes mayores ${ }^{1}$. En la literatura mundial actual la prescripción inadecuada de diuréticos de asa como monoterapia de primera línea en hipertensión, está entre $0,29 \%$ y $3,93 \%$ de todas las PPI según criterios Stopp y Start ${ }^{4-7}$. Por consiguiente, es pertinente identificar su uso como monoterapia en nuestro medio, pues la PPI de fármacos en la población geriátrica se considera una cuestión de salud pública, relacionada con un incremento de la morbilidad, la mortalidad y uso de los recursos sanitarios ${ }^{2}$.

Debe tenerse en cuenta que el sistema de salud colombiano se basa en el aseguramiento global de la población e incluye una serie de procedimientos, tecnologías en salud y medicamentos que están contenidos en un plan de beneficios denominado Plan Obligatorio de Salud, el cual es administrado por una serie de compañías aseguradoras denominadas Entidades Promotoras de Salud (EPS). La furosemida y los demás antihipertensivos están contenidos en dicho plan.

Respecto a la frecuencia de esta PPI, en el país existe un vacío para el cual es importante la realización de esta investigación, de ahí que se buscara identificar la prescripción potencialmente inadecuada de los diuréticos de asa como monoterapia de primera línea en la hipertensión en pacientes mayores de 65 años afiliados al Sistema General de Seguridad Social de Salud de Colombia (SGSSS).

\section{Métodos}

\section{Diseño del estudio}

Se realizó un estudio cuasiexperimental, prospectivo, antes y después, sin grupo control, en el que se llevó a cabo una intervención en médicos tratantes de pacientes mayores de 
65 años de edad con diagnóstico de hipertensión arterial, que estaban en manejo farmacológico con furosemida como monoterapia de primera línea y eran afiliados a diferentes EPS del régimen contributivo del SGSSS durante el $1^{\circ}$. de enero y el 30 de agosto de 2014. Los casos se obtuvieron a partir de la base de datos de Audifarma S.A., un operador logístico encargado de la dispensación institucional de medicamentos en el país, que actualmente distribuye 2 millones de fórmulas al mes. En el departamento de farmacoepidemiología de Audifarma se revisan a diario las estadísticas resultantes de la búsqueda de casos con resultados negativos asociados a la medicación que incluyan problemas de necesidad, efectividad y seguridad.

Se excluyeron todos los sujetos menores de 65 años de edad, que no recibían el fármaco objeto del estudio y que usaran otros medicamentos antihipertensivos (inhibidores del sistema renina-angiotensina-aldosterona (ISRAA), bloqueadores de los canales del calcio, beta-bloqueadores y bloqueantes alfa-1 1 selectivos).

\section{Período de estudio}

En una fase previa a la intervención se identificaron los pacientes con prescripciones de furosemida en monoterapia antihipertensiva durante los tres meses consecutivos anteriores a enero de 2014 , dato que permitió definir el número de casos para llevar a cabo la intervención educativa entre el $1^{\circ}$. de enero y el 30 de agosto de 2014. Los resultados de la intervención se evaluaron entre noviembre de 2014 y febrero de 2015 mediante la determinación de la suspensión de la prescripción del medicamento.

\section{Intervenciones}

Se planteó una intervención que consistió en tres estrategias principales:

1) Reuniones con médicos directores, auditores y líderes de riesgo cardiovascular.

2) Capacitaciones presenciales a médicos prescriptores.

3) Envío de correos electrónicos con bases de datos de pacientes y literatura actualizada del tema.

El objetivo de la intervención era que los médicos responsables de la atención de los pacientes identificados evaluaran la terapia que recibía cada uno y tomara la decisión de retirar la furosemida, o de agregar uno de los medicamentos recomendados en la primera línea de tratamiento antihipertensivo según la valoración clínica de cada caso. Al final, la evaluación del resultado se determinó mediante la cuantificación de la proporción de pacientes sobre los que se modificó el uso de furosemida.

\section{Variables estudiadas}

- Sociodemográficas: edad (anciano joven: 65-74 años; anciano medio: 75-84 años; anciano mayor: 85 o más años), sexo, ciudad de procedencia, EPS.
- Farmacológicas: número mensual de pacientes en tratamiento con furosemida, dosis, comedicación recibida y dosis.

- Cambio del tratamiento por otro antihipertensivo o suspensión del diurético de asa.

\section{Fuentes de información}

La información de la prescripción de furosemida en monoterapia como manejo antihipertensivo, se obtuvo de la base de datos de dispensación de medicamentos de la empresa Audifarma S.A, encargada de la dispensación de fármacos a una población de 6,5 millones de colombianos que corresponde aproximadamente al $14,1 \%$ de la población del país. La detección de los pacientes en terapia con furosemida en monoterapia la realizó un médico epidemiólogo del área de farmacoepidemiología de esta empresa y la información fue auditada por otro médico farmacólogo. Los resultados de la adecuación de la terapia antihipertensiva se obtuvieron relacionando la suspensión en la prescripción del diurético de asa por parte del médico tratante de cada paciente.

\section{Análisis de datos}

La información obtenida se recogió en una base de datos en Excel 2010. Se establecieron frecuencias y proporciones. Para el análisis se empleó el paquete estadístico SPSS 23.0 para Windows. Se hicieron pruebas de $X^{2}$ para las variables categóricas. Se hizo un análisis bivariado considerando como variable dependiente el cambio o la suspensión de la terapia (sí/no) y posteriormente un análisis multivariado con la variable dependiente el mismo cambio de la terapia (sí/no) y como covariables aquellas que se asociaron estadísticamente de manera significativa en los análisis bivariados. Se consideró un valor de $\mathrm{p}<0,05$ como estadísticamente significativo.

\section{Consideraciones éticas}

El estudio recibió el aval del Comité de Bioética de la Universidad Tecnológica de Pereira en la categoría de investigación con riesgo inferior al mínimo, de acuerdo con la Resolución 8430 del Ministerio de Salud de Colombia respetando los principios de confidencialidad de los pacientes establecidos por la Declaración de Helsinki.

\section{Resultados}

Se realizaron un total de 76 actividades, que incluyeron 21 visitas a los responsables de la atención, entre estos, directores médicos y auditores de calidad, en las que se efectuaron 10 sesiones de capacitación con los médicos prescriptores, espacio en el que se les presentó la propuesta, se les dejó material bibliográfico y se resolvieron dudas. Además, de manera periódica, se enviaron 55 correos electrónicos, en los que se reforzaron las intervenciones, con el detalle de cada uno de los pacientes objeto, se justificó la importancia de la modificación de la terapia, previa evaluación del caso, y se aconsejó suspender la furosemida o 
Tabla 1 Variables sociodemográficas de 160 pacientes tratados con furosemida en monoterapia antihipertensiva a partir de un universo de 453.017 sujetos con diagnóstico de hipertensión arterial en Colombia, 2014-2015

\begin{tabular}{lll}
\hline Variables & $\begin{array}{l}\text { Frecuencia } \\
\mathrm{n}=160\end{array}$ & $\%$ \\
\hline Sexo: masculino/femenino & $86 / 74$ & $53,8 / 46,3$ \\
Edad media \pm DE (rango, años) & 82,7 & $66,3-99,0$ \\
Rangos de edad & & \\
Anciano joven 65 a 74 años & 32 & 20,0 \\
Anciano medio 75 a 84 años & 55 & 34,4 \\
Anciano mayor 85 años & 65 & 40,6 \\
Ciudad & & \\
Cali & 39 & 24,4 \\
Bogotá & 21 & 13,1 \\
Manizales & 29 & 18,1 \\
Pereira & 17 & 10,7 \\
Cartagena & 9 & 5,6 \\
Barranquilla & 4 & 2,5 \\
Bucaramanga & 4 & 2,5 \\
Tuluá & 4 & 2,5 \\
Otras 24 ciudades & 33 & 20,5 \\
Entidad aseguradora & & \\
EPS 1 & 70 & 43,8 \\
EPS 2 & 36 & 22,5 \\
EPS 3 & 31 & 19,4 \\
EPS 4 & 19 & 11,9 \\
EPS 5 & 3 & 1,9 \\
EPS 6 & 1 & 0,6 \\
\hline
\end{tabular}

adicionar otro antihipertensivo, con base en la evaluación hecha por el prescriptor.

A partir de un universo de 453.017 sujetos en tratamiento antihipertensivo, se identificaron 160 pacientes con diagnóstico de hipertensión arterial que recibían el diurético de asa en monoterapia, sobre quienes se hizo la notificación de PPI. La edad media fue de 82,7 $\pm 9,1$ años (rango: 66-99 años); la distribución por sexo mostró un ligero predominio de hombres. Las características socio-demográficas de los pacientes se aprecian en la tabla 1. Durante el periodo de observación se halló que en promedio los pacientes recibieron furosemida sin ningún otro antihipertensivo concomitante durante 4 meses. La dosis promedio utilizada fue de $40 \mathrm{mg}$ día, con un rango entre 40 y $160 \mathrm{mg}$ día.

Se logró modificación en la terapia en un total de 67 pacientes (41,8\% de los intervenidos), distribuidos equitativamente entre hombres y mujeres (48 y $52 \%$ respectivamente); todos fueron tratados por médicos de atención primaria.

En 11 pacientes (6,8\% de todos los casos) se suspendió la furosemida y se inició algún otro antihipertensivo; en 21 casos $(13,1 \%)$ se adicionó otro fármaco para el control de la hipertensión arterial, en especial del grupo de ISRAA. A los 35 pacientes restantes $(21,8 \%)$ se les suspendió la furosemida y quedaron sin tratamiento antihipertensivo.

\section{Análisis bivariado}

Se encontró que la variable ser anciano joven se asoció de manera estadísticamente significativa con una mayor probabilidad de éxito en la intervención (OR: 2,2; IC95\%: $1,010-4,860 ; p<0,001)$ ya fuera suspendiendo el diurético de asa y dejando sin tratamiento al paciente o dejando la furosemida y adicionando otro fármaco antihipertensivo; ninguna de las otras variables analizadas se asoció con el éxito de la intervención o la falta de éste.

\section{Discusión}

Se consiguió que los médicos acogieran las recomendaciones y más del $41 \%$ de los pacientes dejaran de recibir un diurético de asa en monoterapia antihipertensiva. Sin embargo, sigue causando preocupación que un número de pacientes continúen recibiéndolo, ya que permanecerán sometidos a un riesgo que está documentado por desequilibrio hidroelectrólítico, alteraciones en el VIII par craneal, e hiperglicemia, entre otras ${ }^{8-10}$. Las razones de la falta de éxito en intervenciones de este tipo pueden explicarse por falta de actualización de los médicos prescriptores, carencia de programas de educación médica permanente y no adherencia a las guías de práctica clínica vigentes que hace bastantes años no incluyen la furosemida como terapia antihipertensiva de primera línea ${ }^{11,12}$.

La furosemida no es considerada medicación antihipertensiva de primera línea y menos aún en monoterapia pues ha sido ampliamente superada por los IECA, Ios BRA-॥, los bloqueadores de los canales del calcio y los diuréticos tiazídicos gracias a su efectividad, comodidad en la administración y perfil de seguridad, y se reserva para el manejo de pacientes con edemas o con hipertensión de difícil control ${ }^{13}$, por lo cual era de esperarse que las recomendaciones hechas a los clínicos fuesen aceptadas y puestas en la práctica en la totalidad de casos.

Pese a que los resultados no consiguieron que los médicos modificaran la formulación en todos los pacientes, es interesante observar que este tipo de intervenciones puede conseguir mejorar las PPI de medicamentos para el tratamiento de una de las patologías más comunes de la práctica clínica, en este caso en adultos mayores de $65 \mathrm{años}^{2,4}$. Otros autores han hecho intervenciones similares y han conseguido correcciones de la formulación hasta en el 93,5\% de pacientes que empleaban broncodilatarores ${ }^{14}$, y este grupo ha obtenido resultados que varían entre el $26 \%$ y el $70 \%$ en pacientes tratados con verapamilo de liberación convencional, o con IECA y BRA-II respectivamente, en quienes se logra la modificación de la terapia ${ }^{15,16}$.

La desprescripción es el acto de reducir o retirar un medicamento (práctica que algunos estudios han demostrado posible y relativamente segura) que clínicamente se pueda considerar innecesario y que incluso pueda mejorar los resultados del manejo de un paciente ${ }^{17,18}$. La detección de PPI se ve apoyada ampliamente con las desprescripciones de aquellos fármacos que por su falta de efectividad, o por sus problemas de seguridad ameritan ser retirados, situación que se consiguió en una proporción de pacientes de este estudio ${ }^{2,4,17}$. Sin embargo, debería esperarse una 
participación más activa de las EPS responsables de los pacientes en ejercer algún tipo de control en la formulación de medicamentos que puedan significar un riesgo importante para estos.

Aunque el número de usuarios de furosemida en monoterapia para el manejo de la hipertensión arterial es bajo, los resultados sugieren la necesidad de reforzar la formación de los médicos a través de estrategias de educación continuada que aborden el tema del uso de medicamentos que fueron superados por otras opciones terapéuticas, más convenientes y seguras ${ }^{13}$.

Este estudio cuenta con algunas limitaciones relacionadas con el origen de la información que proviene de una base de datos de medicamentos dispensados y no de la historia clínica o de entrevistas con el paciente o su prescriptor. De igual forma no es posible garantizar que todos los medicamentos reclamados, fueron consumidos por el paciente, como tampoco se conoció qué otras terapias recibía el paciente por fuera de su plan de salud. Además, no se puede asegurar que en todos los casos que se presentó la suspensión o el cambio del medicamento se debió a la intervención realizada por los investigadores, y por el diseño del trabajo no se puede determinar que otros factores hayan influido en el resultado.

No obstante, la fortaleza del estudio radica en que logró identificar una cohorte de pacientes con diagnóstico de hipertensión arterial que estaba recibiendo PPI con furosemida en monoterapia y se consiguió llegar hasta los médicos responsables de la atención sanitaria para que evaluaran las recomendaciones y decidieran sobre la continuidad del tratamiento. Esto arrojó como resultado la desprescripción del diurético en una proporción importante de $\operatorname{casos}^{17,18}$. Además, se hizo una revisión cuidadosa de la información de seguimiento de los medicamentos que continuaron recibiendo los pacientes.

Se puede concluir que una pequeña proporción de pacientes colombianos mayores de 65 años con hipertensión arterial siguen siendo tratados con furosemida como único fármaco, a pesar de que no está recomendado por las guías actuales de tratamiento y existen evidencias de su menor efectividad comparada con otros grupos de antihipertensivos y de sus problemas de seguridad; no obstante, mediante la utilización de estrategias educativas y de seguimiento de las prescripciones se puede reducir su uso inapropiado. La única variable que se asoció con mayor probabilidad de éxito en la intervención fue pertenecer al grupo de ancianos entre 65 y 74 años. Es importante desarrollar herramientas que incentiven al médico a seguir las indicaciones propuestas para disminuir las PPI. Todos los responsables de la cadena del medicamento, incluidos los operadores logísticos que los dispensan, deben realizar seguimiento de las prescripciones para determinar su seguridad y la menor cantidad de riesgos para el paciente.

\section{Responsabilidades éticas}

Protección de personas y animales. Los autores declaran que los procedimientos seguidos se conformaron a las normas éticas del comité de experimentación humana responsable y de acuerdo con la Asociación Médica Mundial y la Declaración de Helsinki.
Confidencialidad de los datos. Los autores declaran que han seguido los protocolos de su centro de trabajo sobre la publicación de datos de pacientes.

Derecho a la privacidad y consentimiento informado. Los autores han obtenido el consentimiento informado de los pacientes y/o sujetos referidos en el artículo. Este documento obra en poder del autor de correspondencia.

\section{Financiación}

El trabajo recibió financiación de la Universidad Tecnológica de Pereira y Audifarma S.A.

\section{Conflictos de interés}

Los autores declaran no tener conflictos de interés.

\section{Agradecimientos}

A Soffy López, Viviana Orozco y Andrea Orozco por su colaboración en la generación de la base de datos.

\section{Bibliografía}

1. Delgado Silveira E, Munoz Garcia M, Montero Errasquin B, Sanchez Castellano C, Gallagher PF, Cruz-Jentoft AJ. Inappropriate prescription in older patients: the STOPP/START criteria. Rev Esp Geriatr Gerontol. 2009;44:273-9.

2. Gallagher P, Ryan C, Byrne S, Kennedy J, O'Mahony D. STOPP (Screening Tool of Older Person's Prescriptions) and START (Screening Tool to Alert doctors to Right Treatment). Consensus validation. Int J Clin Pharmacol Ther. 2008;46:72-83.

3. James PA, Oparil S, Carter BL, Cushman WC, DennisonHimmelfarb C, Handler J, et al. 2014 evidence-based guideline for the management of high blood pressure in adults: report from the panel members appointed to the Eighth Joint National Committee (JNC 8). JAMA. 2014;311:507-20.

4. Hamilton H, Gallagher P, Ryan C, Byrne S, O'Mahony D. Potentially inappropriate medications defined by STOPP criteria and the risk of adverse drug events in older hospitalized patients. Arch Intern Med. 2011;171:1013-9.

5. Ryan C, O'Mahony D, Kennedy J, Weedle P, Byrne S. Potentially inappropriate prescribing in an Irish elderly population in primary care. Br J Clin Pharmacol. 2009;68:936-47.

6. Vezmar Kovacevic S, Simisic M, Stojkov Rudinski S, Culafic $M$, Vucicevic K, Prostran $M$, et al. Potentially inappropriate prescribing in older primary care patients. PLoS One. 2014;9: e95536.

7. Bradley MC, Motterlini N, Padmanabhan S, Cahir C, Williams T, Fahey T, et al. Potentially inappropriate prescribing among older people in the United Kingdom. BMC Geriatr. 2014;14:72.

8. Farcas A, Bucsa C, Sinpetrean A, Leucuta D, Mogosan C, Dumitrascu $D$, et al. Preventability analysis of adverse drug reactions detected in two internal medicine departments in Romania. Intern Emerg Med. 2014;9:187-93.

9. Marusic S, Sicaja M, Obreli Neto PR, Franic M, Marinovic I, Bacic-Vrca V. Adverse drug reactions in elderly patients following discharge from an internal medicine clinic. Int $\mathrm{J}$ Clin Pharmacol Ther. 2014;52:906-13.

10. Keller GA, Di Girolamo G, Alvarez PA. Pharmacovigilance and the cardiovascular system: two sides to every story. Curr Drug Saf. 2011;6:224-9. 
11. Theodorou M, Stafylas P, Kourlaba G, Kaitelidou D, Maniadakis $\mathrm{N}$, Papademetriou V. Physicians' perceptions and adherence to guidelines for the management of hypertension: a national, multicentre, prospective study. Int J Hypertens. 2012;2012:503821.

12. Taddei S, Bruno RM, Ghiadoni L. The correct administration of antihypertensive drugs according to the principles of clinical pharmacology. Am J Cardiovasc Drugs. 2011;11:13-20.

13. Baguet JP, Robitail S, Boyer L, Debensason D, Auquier P. A meta-analytical approach to the efficacy of antihypertensive drugs in reducing blood pressure. Am J Cardiovasc Drugs. 2005;5:131-40.

14. Fernandez Urrusuno R, Perez Perez P, Montero Balosa MC, Gonzalez Limones S, Caraballo Camacho MO, Cuberos Fernandez V. Quality prescribing of long-acting beta-agonists. Results of an intervention in Primary Care. Rev Calid Asist. 2012;27:11-8.
15. Machado-Alba JE, Giraldo-Giraldo C, Machado-Duque ME. Quality of conventional release verapamil prescription in patients with arterial hypertension. Rev Calid Asist. 2015;30: 72-8.

16. Portilla A, Torres D, Machado-Duque ME, Machado-Alba JE. Intervención en la prescripción de la combinación de inhibidor de la enzima convertidora de angiotensina (IECA) y bloqueador del receptor de angiotensina II (BRA-II). Aten Primaria. 2016;48:272-4.

17. Farrell B, Tsang C, Raman-Wilms L, Irving H, Conklin J, Pottie $\mathrm{K}$. What are priorities for deprescribing for elderly patients? Capturing the voice of practitioners: a modified delphi process. PLoS One. 2015; 10:e0122246.

18. Scott IA, Hilmer SN, Reeve E, Potter K, Le Couteur D, Rigby $D$, et al. Reducing inappropriate polypharmacy: the process of deprescribing. JAMA Intern Med. 2015;175:827-34. 